\title{
SORÇÃO DE ATRAZINA EM CAMBISSOLO HÚMICO DO RIO GRANDE DO SUL SOB VEGETAÇÃO NATIVA ${ }^{(1)}$
}

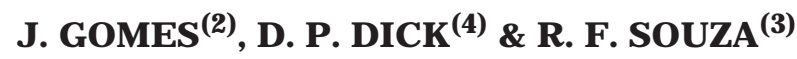

\begin{abstract}
RESUMO
A acumulação de her bicidas no ambiente em virtude de sua larga utilização em sistemas agrícolas, associada à alta persistência, é extremamente preocupante, considerando os efeitos maléficos que alguns destes compostos causam à flora e à fauna. A atrazina (2-cloro-4-etilamino-6-isopropilamino-striazina) é um dos herbicidas mais utilizados na atualidade e tem sido detectada em teores consideráveis em mananciais e solos. O objetivo deste estudo foi avaliar o comportamento sortivo da atrazina comercial em Cambissolo Húmico em condições naturais e em ausência de matéria orgânica. Foram determinadas isotermas de sorção de atrazina comercial em amostras do horizonte A na sua forma natural e na forma oxidada. A quantidade máxima de herbicida sorvido variou de $8 \%$ (amostra oxidada) a $49 \%$ (amostra natural) da quantidade adicionada. A aplicação do modelo de Freundlich na forma linear aos dados experimentais forneceu altos coeficientes de correlação para a sorção em amostra natural $(r=0,960, P<0,01)$ e na amostra oxidada $(r=0,937, P<0,01)$. O coeficiente $\mathrm{n}_{\mathrm{f}}$ (modelo de Freundlich) obtido na amostra natural $(1,40)$ indica que a afinidade do sorbato pelo sorvente aumentou com o progresso da sorção, enquanto, na amostra oxidada, o comportamento foi inverso $\left(n_{f}=0,78\right)$. $O$ valor do coeficiente $\mathrm{K}_{\mathrm{f}}$ foi de $1,10 \mathrm{~L} \mathrm{~kg}^{-1}$ na amostra natural e de $0,84 \mathrm{~L} \mathrm{~kg}^{-1}$ na amostra oxidada, enquanto o coeficiente de distribuição da atrazina $\left(K_{d}\right)$ foi de 4,64 e 0,33 $\mathrm{L} \mathrm{kg}^{-1}$, respecti vamente. Estes resultados mostram que a matéria orgânica foi o sorvente determinante na retenção de atrazina no Cambissolo húmico. Adicionalmente, o valor de $\mathrm{K}_{\mathrm{oc}}$ de $103 \mathrm{~L} \mathrm{~kg}^{-1}$ obtido classifica a atrazina comercial como de alta mobilidade para o sistema atrazina-solo estudado.
\end{abstract}

Termos de indexação: herbicida, Freundlich, matéria orgânica, afinidade química.

\footnotetext{
(1) Trabalho apresentado no X Congresso da IHSS, julho de 2000, Toulouse, França. Submetido em outubro de 2000 . Recebido para publicação em novembro de 2000 e aprovado em outubro de 2001.

(2) Mestranda em Ciência do Solo, Faculdade de Agronomia, Universidade Federal do Rio Grande do Sul - UFRGS. Av. Bento Gonçalves 9500, CEP 91501-970 Porto Alegre (RS). E-mail: juliana@epovo.net

(3) Professor do Departamento de Físico-Química, Instituto de Química, UFRGS. E-mail: rfds@iq.ufrgs.br

(4) Professora do Departamento de Ciência doSolo, Faculdade deAgronomia, UFRGS. Bolsista do CNPq. E-mail: dpdick@hotmail.com
} 


\title{
SUMMARY: ADSORPTION OF ATRAZINE ON A HUMIC CAMBISOL FROM RIO GRANDE DO SUL UNDER NATIVE VEGETATION
}

\begin{abstract}
Theaccumulation of herbicides in theenvironment as a consequence of their intensive usageassociated to their high persistanceis a matter of principal concern duetotheir harmful effects on thefauna and flora. Atrazi ne(2-chloro-4-ethylami no-6-isopropyl amino-s-triazine) is oneof theworldwidemostly used herbicides, and is being detected in considerableamounts in aquatic and soil ecosystems. Thegoal of this study was to eval uatethesorptive behavi our of atrazine in a Humic Cambisol under its natural form and in the absence of organic matter. Sorption isotherms of commercial atrazine were determined in samples of an A horizon in its natural state and in an oxidized sample. The maximal quantity of sorbed herbicideranged from $8 \%$ (oxidized sample) to $49 \%$ (natural sample) of theadded amount. The Freundlich model in the linear form was applied to the experimental data and high correlation coefficients were obtained in both the natural sample $(R=0.960, P<0.01)$ and theoxi dized sample $(R=0.932, P<0.01)$. The F reundlich coefficient $\left(n_{f}=1.40\right)$ obtained in the natural samples indicates that the sorbate affinity for the sorbent increased with the adsorption increment, while in the oxidized sample the opposite behaviour was observed $\left(n_{f}=0.78\right)$. The Freundlich coeficient $K_{f}$ was $1.10 \mathrm{~L} \mathrm{~kg}^{-1}$ for the natural sample and $0.84 \mathrm{~L} \mathrm{~kg}^{-1}$ for the oxidized sample, and the distribution coefficient $\mathrm{Kd}$ ranged from 4.64 to $0.33 \mathrm{~L} \mathrm{~kg}^{-1}$, respectivel $y$. These results show that the organic matter was the most relevant atrazinesorbent in theH umic Cambisol. Furthermore, the $\mathrm{K}_{\text {oc }}$ value of $103 \mathrm{~L} \mathrm{~kg}^{-1}$, classifies the commercial atrazine as of high mobility in the studied soil-atrazine system.
\end{abstract}

Index terms: herbicide, Freundlich, organic matter, chemical affinity.

\section{NTRODUÇÃO}

A atrazina (2-cloro-4-etilamino-6-isopropilaminos-triazina) foi introduzida na agricultura na década de 50 e é atualmente o herbicida mais utilizado no mundo: 70.000 a 90.000 toneladas anuais (Steinberg et al., 1995; Graymoreet al., 2001). Em geral, apenas parte da quantidade aplicada de um herbicida é bioativa, sendo o restante distribuído no ambiente (Lagaly, 2001). Para a atrazina, o tempo de meiavida pode variar de alguns meses a seis anos (Armstrong et al., 1967).

A detecção deste herbici da e de seus metabólitos em lençóis freáticos e mananciais de países como Alemanha eSuécia, onde sua utilização foi proi bida na década de 90 , mostra o alto poder contaminante a longo prazo desses compostos (Kruger et al., 1997; Graymore et al., 2001). Nos Estados Unidos, a concentração de atrazina detectada em Iençóis freáti cos chega a ser 20 vezes superior a de qual quer outro herbicida (Belluck et al., 1991). Essa el evada persistência éextremamente preocupante por causa de seu efeito carcinogênico (Patlack, 1996) e de seu poder de desencadear al terações cromossômicas que podem levar à má-formação congênita (Biradar \& Rayburn, 1995).

Processos de sorção desempenham importante papel na dinâmica de um herbicida no ambiente, uma vez que regulam a disponibildade do composto para ser degradado, volatilizado, lixiviado e absorvido pelas plantas (Herwig et al., 2001). Esses processos, por sua vez, dependem das características do solo, tais como: teor e qualidade de matéria orgânica, teor etipo de minerais, capacidade detroca catiônica (CTC), pH, umidadeetemperatura (Kruger et al., 1997; Rodrigues \& Almeida, 1998).

A contribuição da fração mineral na retenção de atrazina em sol os é moderada, quando comparada com o poder sorvente da matéria orgânica (Barriuso \& Calvet, 1992; Laird et al ., 1994; Celis et al., 1997; Queiroz \& Lanças, 1997; J avaroni et al., 1999). A sorção de atrazina na superfície de esmectitas e caulinitas ocorre principalmente por pontes de hidrogênio e por interações hidrofóbicas em superfícies de baixa densidade de carga (Laird et al., 1992; Sawhney \& Singh, 1997; Herwig et al., 2001).

Martin-Neto et al. (1994), por meio de técnicas espectroscópicas, mostraram queas interações entre ácidos húmi cos extraídos de um Oxissolo brasileiro ea atrazina ocorrem por pontes de hidrogênio e por ligações hidrofóbicas. Resultados semel hantes foram obtidos por Senesi \& Testini (1982). Piccolo et al. (1998) constataram a contribuição rel evante dos grupamentos alifáticos no processo de sorção e concluíram que a interação hidrofóbica constitui importante mecanismo na reação de atrazina com substâncias húmicas. Recentemente, Martin-Neto et al. (2001), corroborando estes resultados, também 
verificaram que a hidróxi-atrazina, metabólito da atrazina cuja formação pode ser catalisada por substâncias húmicas, sorve também por meio de mecanismo de transferência de elétrons.

No Brasil, a atrazina é aplicada principalmente nas plantações de milho em pré-emergência, sendo utilizada também em culturas de cana-de-açúcar e sorgo (Rodrigues \& Almeida,1998). Ainda que existam vários estudos na literatura sobre a interação de atrazina com matéria orgânica, com substâncias húmicas e com minerais (Xing et al., 1996; Celis et al., 1997; Herwig et al., 2001; MartinNeto et al., 2001), trabalhos sobre a dinâmica da atrazina em solos e, particularmente, em solos brasileiros, são escassos (Albuquerque et al., 2001).

O objetivo deste trabalho foi avaliar o comportamento sortivo da atrazina em Cambissolo Húmico do Rio Grande do Sul, em presença e em ausência da matéria orgânica original, por meio da determinação de isotermas de sorção do herbicida.

\section{MATERIAL E MÉTODOS}

\section{Sorvente}

O sorvente empregado foi o horizonte A de um Cambissolo Húmico (Bom J esus, RS), cuja mineralogia é composta principalmente por: caulinita $\left(\mathrm{Al}_{2}(\mathrm{OH})_{4} \mathrm{Si}_{2} \mathrm{O}_{5}\right)$, goethita $(\alpha-\mathrm{FeOOH})$, hematita $\left(\alpha-\mathrm{Fe}_{2} \mathrm{O}_{3}\right)$ e quartzo $\left(\mathrm{SiO}_{2}\right)$. O solo foi utilizado na forma natural e na forma oxidada.

\section{Sorbato}

Utilizou-se como sorbato o herbicida comercial atrazina (N ovartis, $500 \mathrm{~g} \mathrm{~L}^{-1}$ de ingrediente ativo).

\section{Preparação dos sorventes}

A amostra natural foi seca a $60^{\circ} \mathrm{C}$ e triturada em gral de ágata. Para remover a matéria orgânica, foram tratados $30 \mathrm{~g}$ de solo com $60 \mathrm{~mL}$ de solução de $\mathrm{H}_{2} \mathrm{O}_{2} / \mathrm{H}_{2} \mathrm{O}(15 / 85, \mathrm{v} / \mathrm{v})$ a $60^{\circ} \mathrm{C}$, durante dois períodos de $10 \mathrm{~h}$, sob agitação periódica com bastão de vidro. A solução oxidante foi sendo reposta conforme ocorria evaporação. Após o tratamento, o sol ofoi lavado com $30 \mathrm{~mL}$ deágua destilada (2 vezes), seco em estufa a $60^{\circ} \mathrm{C}$ e triturado em gral de ágata.

\section{Caracterização dos sorventes}

O teor de carbono nos sorventes foi determinado por combustão seca (analisador elementar Perkin EImer 2400). A caracterização por Espectroscopia no Infravermelho por Refletância Difusa com Transformada deFourier (DRIFT) (espectrofotômetro Bomem MB 102) foi realizada em amostras em pó, varrendo-se o espectro de 4.000 a $400 \mathrm{~cm}^{-1}$, com um acúmulo de 300 scans e resolução de $4 \mathrm{~cm}^{-1}$.

A determinação da área superficial específica (ASE ) foi efetuada com três repetições pelo método da sorção de água a $20 \%$ de umidade relativa (UR) (Quirk, 1955). Cerca de 2,00 g de solo (exatamente pesados) foram colocados em frascos pesa-filtros e desidratados em dessecador sob $\mathrm{P}_{2} \mathrm{O}_{5}$, durante 10 dias, a $25^{\circ} \mathrm{C}$. Após pesagem, as amostras foram colocadas no dessecador com solução saturada de acetato de potássio (M erck) (20\% deUR), e o sistema foi fechado por mais 10 dias. A amostra foi então novamente pesada. Considerou-se que a $20 \%$ de UR, a água adsorve na fase sólida numa camada monomolecular, ocupando cada molécula de água uma área de 0,108 $\mathrm{nm}^{2}$. Portanto, $1 \mathrm{mg}$ de água adsorvida a $20 \%$ de UR corresponde a uma área de recobrimento de 3,6 $\mathrm{m}^{2}$.

A determinação do $\mathrm{pH}$ dos sorventes foi feita em água destilada $\left(\mathrm{pH}_{\mathrm{H}_{2} \mathrm{O}}\right)$ e em solução de $\mathrm{KCl} 1 \mathrm{~mol} \mathrm{~L}^{-1}$ $\left(\mathrm{pH}_{\mathrm{KCl}}\right)$, empregando-se $10 \mathrm{~cm}^{-3}$ de solo e $25 \mathrm{~mL}$ de solu ução (EMBRAPA, 1997). A partir destes valores, foi cal culado o $\Delta \mathrm{pH}\left(\mathrm{pH}_{\mathrm{KCl}}-\mathrm{pH}_{\mathrm{H}_{2} \mathrm{O}}\right)$.

\section{Preparação e caracterização do sorbato}

Foram preparadas soluções de atrazina com concentrações de8, 10, 16, 26 e $30 \mathrm{mg} \mathrm{L}^{-1}$. Determinouseo pH eajustou-se a força iônica a 0,01 mol L-1 com solução de $\mathrm{CaCl}_{2}$ (Merck). As soluções foram guardadas na geladeira eno escuroaté sua utilização. O espectro da solução de atrazina $8 \mathrm{mg} \mathrm{L}^{-1}$ foi determinadona regiãoUV/visível (E spectrofotômetro Shimadzu 150-A).

\section{Determinação das isotermas de sorção}

As reações de sorção foram realizadas em temperatura ambiente $\left(20-25^{\circ} \mathrm{C}\right)$, empregando-se $2,00 \mathrm{~g}$ de sol o e $20 \mathrm{~mL}$ de solução deatrazina a pH 6 . Após seis horas de agitação no escuro, a solução de atrazina foi separada do solo por centrifugação, durante $10 \mathrm{~min}$ (3.000 rpm), seguida de filtração (Whattman 45), e o pH das soluções foi medido. O procedimento foi feito em duplicata e com acompanhamento do branco (solução de $\mathrm{CaCl}_{2}$ $\left.0,01 \mathrm{~mol} \mathrm{~L}^{-1}\right)$. As soluções de atrazina foram guardadas em gel adeira e no escuro até à real ização da análise por cromatografia líquida de alta performance (HPLC).

A concentração de atrazina em solução, antes e depois da sorção, foi determinada por HPLC (LDC Analytical -Consta M etric 3200), com detector UV a $222 \mathrm{~nm}$ (LDC Analytical Spectra Monitor 3200), empregando coluna de fase reversa RP-18 (Spheri5) $(250 \times 4,6 \mathrm{~mm})$. A fase móvel utilizada foi a sol ução metanol/água (70/30, v/v), o vol ume injetado foi de $20 \mu \mathrm{L}$, fluxo de $0,5 \mathrm{~mL} \mathrm{~min}^{-1}$ efiltração prévia em membrana Millipore 0,45 $\mu \mathrm{m}$. A quantidade adsorvida foi obtida em função da variação da área do pico correspondente à atrazina:

$$
\% \text { ads }=\frac{\left(\mathrm{A}_{0}-\mathrm{A}_{\mathrm{t}}\right) 100}{\mathrm{~A}_{\mathrm{t}}}
$$


em que

$\%_{\text {ads }}=$ percetagem de atrazina sorvida

$\mathrm{A}_{0}=$ área correspondente à atrazina antes da sorção

$A_{t}=$ área da atrazina em solução após sorção.

Conhecendo a massa inicial, calcul ou-se a massa de atrazina sorvida:

$$
\mathrm{Q}_{\mathrm{ads}}=\frac{\% \operatorname{ads} \times \mathrm{M}_{\mathrm{i}}}{100} \cdot \frac{1}{\mathrm{M}_{\text {solo }}}
$$

em que

$\mathrm{Q}_{\mathrm{ads}}=$ quantidade de atrazina sorvida $\left(\mathrm{mg} \mathrm{kg}^{-1}\right)$

$M_{i}=$ massa de atrazina em solução antes da sor ção (mg)

$\mathrm{M}_{\text {solo }}=$ massa de sorvente (solo) utilizada na sorção $(\mathrm{kg})$.

O val or de \% ads, empregado no cál culo de $\mathrm{Q}_{\text {ads, }}$, corresponde à média aritmética das duas determinações cromatográficas.

Previamente à determinação das isotermas de sorção, foi feita uma curva de calibração, para verificar a correlação linear entre a área do pico correspondente à atrazina na quantificação por HPLC e a massa do herbicida em solução, no intervalo de concentração utilizado.

\section{Tratamento matemático}

Aos dados experimentais foi aplicada a isoterma de Freundlich na forma linear:

$$
\ln \mathrm{Q}_{\mathrm{ads}}=\ln \mathrm{K}_{\mathrm{f}}+\mathrm{n}_{\mathrm{f}} \ln \mathrm{C}_{\mathrm{eq}}
$$

em que

$\mathrm{C}_{\text {eq }}=$ concentração de atrazina na solução de equilíbrio ( $\mathrm{mg} \mathrm{L}^{-1}$ )

$\mathrm{K}_{\mathrm{f}}=$ constante relativa ao sistema atrazina-solo $\left(\mathrm{L} \mathrm{kg}^{-1}\right)$

$\mathrm{n}_{\mathrm{f}}=$ constante relativa à inclinação da curva.

O coeficiente de distribuição $K_{d}\left(L_{k g}{ }^{-1}\right)$ para o sistema solo-solução de atrazina após sorção foi calculado por regressão linear, assumindo $n_{f}=1$. 0 coeficiente de distribuição em relação ao carbono orgânico do solo, $\mathrm{K}_{\mathrm{OC}}\left(\mathrm{L} \mathrm{g}^{-1}\right)$ foi cal culado segundo:

em que

$$
\mathrm{K}_{\mathrm{oc}}=\left(\mathrm{K}_{\mathrm{d}} / \mathrm{C}\right) .1000
$$

$\mathrm{C}=$ teor de carbono orgânico do solo $\left(\mathrm{g} \mathrm{kg}^{-1}\right)$

\section{RESULTADOS E DISCUSSÃO}

\section{Características dos sorventes}

Ambos os sorventes apresentaram valores de pH em água levemente ácidos (Quadro 1). Os valores de $\Delta \mathrm{pH}$ calculados foram negativos, indicando que, nos dois sorventes, ocorreu carga líquida de superfície negativa (Mekaru \& Uehara, 1972). Adicional mente, o menor val or numérico de $\Delta \mathrm{pH}$ da amostra oxidada mostra que a remoção da matéria orgânica causou uma diminuição da carga negativa líquida. A contribuição importante dos grupos carboxilatos (R-COO-) na CTC (Sparks, 1995) fica evidenciada por este resultado, uma vez que estes grupos funcionais estão ausentes na amostra oxidada, conforme resultados de espectroscopia de DRIFT (veja discussão mais adiante).

A remoção da matéria orgânica, no entanto, não foi completa, uma vez que $6,6 \mathrm{~g} \mathrm{~kg}^{-1}$ de $C$ resistiram à oxidação (Quadro 1). O tratamento com peróxido de hidrogênio também causou a diminuição da ASE (Quadro 1), o que está relacionado com o fato de os minerais caul inita, goethita ehematita apresentarem ASE inferior à da matéria orgânica (Sparks, 1995). A remoção da matéria orgânica pode causar dois efeitos contrários na ASE: (a) aumento da ASE mediante a destruição de microagregados e (b) diminuição da ASE mediante a retirada de um sorvente de alta área superficial. No presente estudo, o segundo efeito parece ter predominado sobre o primeiro.

Os espectros da amostra natural e da oxidada apresentaram diferenças, principalmente na região de 1.700 a $1.250 \mathrm{~cm}^{-1}$ (Figura 1). Após o solo ser tratado com peróxido de hidrogênio (espectro $B$ ), a banda larga em $1.378 \mathrm{~cm}^{-1}$ e a banda em $1.632 \mathrm{~cm}^{-1}$ do espectro A, correspondentes aos grupos carboxilatos de superfície (Dick et al., 2000), desapareceram devido remoção da matéria orgânica. Nessa região, apareceram dois picos de menor intensidade em 1.423 e $1.299 \mathrm{~cm}^{-1}$ atribuídos a minerais (Farmer, 1974; Tan, 1996) (absorções inespecíficas), que, no sol o natural, se encontravam encobertos pela matéria orgânica. A banda próxima a $1.673 \mathrm{~cm}^{-1}$ no espectro $B$ foi atribuída ao grupo $\mathrm{OH}$ da água estrutural dos minerais (Farmer, 1974) e estava encoberta pela banda em $1.634 \mathrm{~cm}^{-1}$ que apresentava maior intensidade. Bandas de absorção próximas a $2.900 \mathrm{~cm}^{-1}$, atribuídas aos grupos $\mathrm{C}-\mathrm{H}$ alifáticos (Dick et al., 2000), desapareceram após a oxidação do solo. Comportamento semelhante,

Quadro 1. Atributos do Cambissolo Húmico na forma natural e oxidada

\begin{tabular}{lcc}
\hline & \multicolumn{2}{c}{ Amostra } \\
\cline { 2 - 3 } Atributo & Natural & Oxidada \\
\hline $\mathrm{pH}_{\mathrm{H}_{2} \mathrm{O}}$ & 5,01 & 5,23 \\
$\mathrm{pH} \mathrm{KCl}$ & 3,99 & 4,55 \\
$\Delta \mathrm{pH}$ & $-1,02$ & $-0,68$ \\
$\mathrm{C}\left(\mathrm{mg} \mathrm{g}^{-1}\right)$ & 45,2 & 6,6 \\
$\mathrm{ASE}\left(\mathrm{m}^{2} \mathrm{~g}^{-1}\right)$ & 100 & 75 \\
\hline
\end{tabular}


decorrente da destruição da matéria orgânica pelo tratamento com $\mathrm{H}_{2} \mathrm{O}_{2}$, foi observado por outros autores (Laird et al., 1994).

Nos dois espectros (Figura 1), observaram-se picos finos em 3.698, 3.624 e $3.650 \mathrm{~cm}^{-1}$ ocorridos devido aos grupos $\mathrm{Al}-\mathrm{OH}$ e $\mathrm{Si}-\mathrm{OH}$ de superfície do mineral caulinita (Tan, 1996). A banda larga em $3.453 \mathrm{~cm}^{-1}$ corresponde a grupos $\mathrm{OH}$ em ponte. As absorções em 1.103 eem $1.160 \mathrm{~cm}^{-1}$ foram atribuídas aos grupos Si-O (Farmer, 1974).

\section{Caracterização do sorbato}

O espectro da solução de atrazina $\left(8 \mathrm{mg} \mathrm{L}^{-1}\right)$ na região do UVNis apresentou um máximo deabsorção em $222 \mathrm{~nm}$, que é o comprimento de onda usualmente utilizado para a quantificação da atrazina quando se usa detector de UV (Barriuso et al., 1992; Martin-N eto et al., 1994). A ausência de um pico em $240 \mathrm{~nm}$ comprovou que as soluções empregadas nos testes de sorção não continham hidróxi-atrazina.

\section{I sotermas de sorção}

A técnica de HPLC mostrou-se eficaz e rápida para a quantificação da atrazina. As condições utilizadas permitiram a separação da atrazina de outros compostos eventualmente extraídos do solo, conforme comprovado pel o teste em branco.

As isotermas de sorção elaboradas a partir dos dados experimentais encontram-se na figura 2. Em nenhum dos sorventes foi obtida a sorção máxima, pois as condições experimentais empregadas (razão sólido:solução) não foram adequadas para saturar todos os sítios sorventes, tendo em vista que a solubilidade máxima da atrazina em água é $30 \mathrm{mg} \mathrm{L}^{-1}$. Na amostra oxidada, a quantidade máxima de herbicida sorvido foi $8 \%$ da quantidade adicionada, enquanto, na amostra natural, obtevese um máximo de $49 \%$ de herbicida sorvido para as mesmas condições experimentais. I sto comprova a alta capacidade de sor ção da matéria orgânica neste solo. Em um Latossolo Roxo (L atossolo Vermelho, segundo EMBRAPA (1999)), foram obtidos resultados semelhantes: nesse solo, a matéria orgânica sorveu 50 \% da atrazina adicionada e a fração mineral apenas 5 \% (Albuquerque et al., 2001).

A aplicação da equação de Freundlich aos dados experimentais forneceu altos coeficientes de correlação: $r=0,960$ ( $P<0,01)$, para o sol o natural, e $r=0,937$ ( $P<0,01)$, para a amostra oxidada (Figura 2).

A equação de Freundlich é um modelo empírico e sua adequação a dados experimentais é usualmente relacionada com a existência de forças físicas que envolvem baixas energias de sorção, ocorrendo formação de múltiplas camadas (Sparks, 1995; Atkins, 1999). O comportamento verificado no presente trabal ho está de acordo com resultados obtidos por outros autores, que observaram que a interação entrea matéria orgânica eatrazina ocorre principalmente por interações físicas, tais como: pontes de hidrogênio (Martin-Neto et al., 1994) e interações hidrofóbicas (Picoll lo et al., 1998; MartinNeto et al., 2001).

A equação exponencial da isoterma com expoente $>1$ obtida no solo natural $\left(n_{f}=1,40\right)$ é usualmente atribuída ao aumento da afinidade do sorbato pelo sorvente, conforme progride a sor ção (Sparks, 1995).

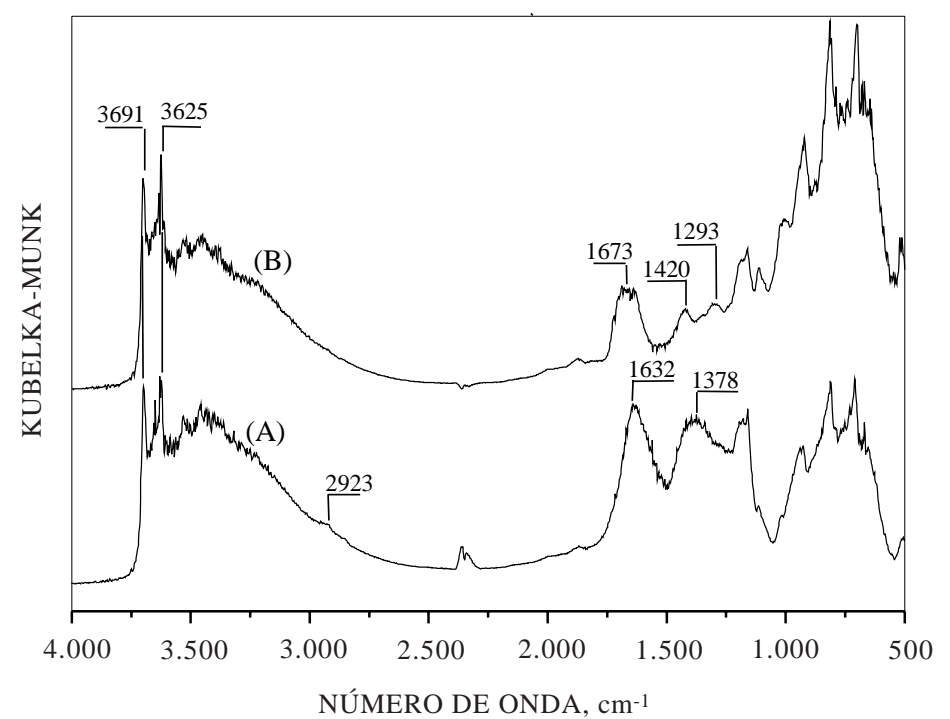

Figura 1. Espectros de DRIFT do Cambissolo Húmico na forma natural (A) e oxidada (B). 


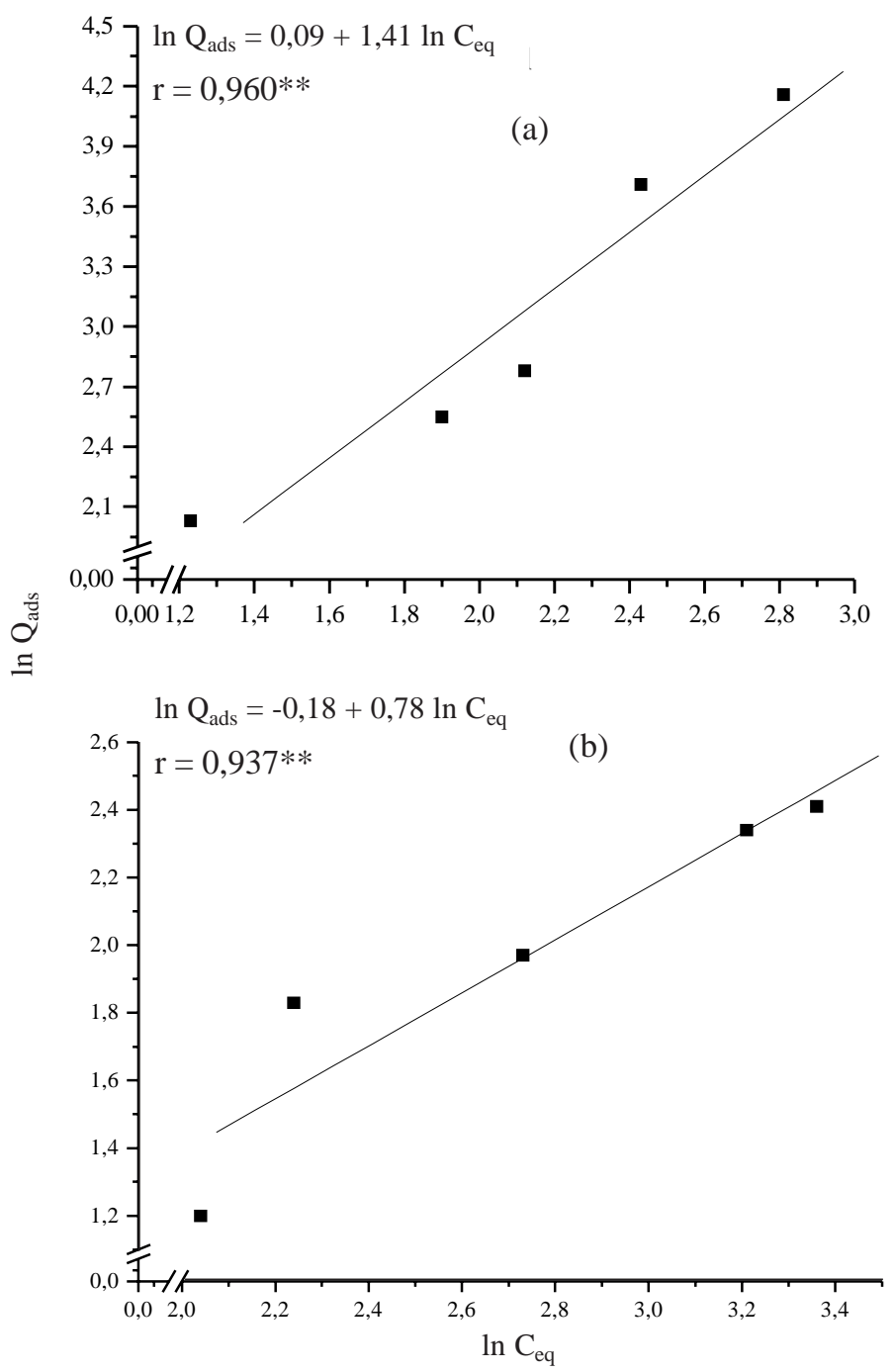

Figura 2. Isoterma de sorção de atrazina em Cambissolo Húmico na forma natural (a) e oxidada (b). $Q_{\text {ads: }}$ quantidade de atrazina sorvida, $\mathrm{mg} \mathrm{kg}^{-1} ; \mathrm{C}_{\text {eq }}$;concentração de atrazi na em equilíbrio, $\mathrm{mg} \mathrm{L}^{-1}$; $* *$ : $P<0,01$.

No caso de sorção de compostos orgânicos pelo solo, este aumento de afinidade pode ser relacionado com interações cooperativas entre moléculas sorvidas, estimulando a sorção posterior do sorbato (Sposito, 1989). Para a sorção da atrazina na amostra oxidada, que revela baixa afinidade herbicida-sorvente (Laird et al., 1994; Xing et al., 1996; Celis et al., 1997), este efeito cooperativo deveria ser mais pronunciado. No entanto, foi obtida uma equação com expoente $<1$ $\left(n_{f}=0,78\right)$, o que caracteriza uma diminuição da afinidade com o aumento da sorção.

O comportamento observado na amostra natural pode estar relacionado com a formulação da atrazina comercial empregada neste estudo, que pode conter algum componente responsável pelo aumento da afinidade do sorbato pelo sorvente com o progresso da sorção. Todavia, esta hipótese não pode ser testada neste estudo.
Queiroz \& Lanças (1997) obtiveram para a sorção de atrazina em cinco sol os de São Paulo val ores de $n_{f}$ entre 0,88 e 1,28, sendo que o sol o com menor teor decarbono orgânico $\left(8,0 \mathrm{~g} \mathrm{~kg}^{-1}\right)$ apresentou o menor valor de $\mathrm{n}_{\mathrm{f}}$ do grupo estudado. Em contrapartida, em solos cauliníticos e gibbsíticos com teores de matéria orgânica de 27 a $40 \mathrm{~g} \mathrm{~kg}^{-1}$, valores de $\mathrm{n}_{\mathrm{f}}$ situaram-se em torno de 1,1 (Vaz et al., 1997).

O coeficiente $K_{f}$, obtido pela equação linearizada de Freundlich, foi mais el evado no solo natural, o que indica uma diminuição da retenção da atrazina com a remoção da matéria orgânica do sistema (Quadro 2). Comportamento semelhante foi observado por Moreau \& Mouvet (1997) em um Planossolo, que obtiveram um valor de $3,1 \mathrm{~L} \mathrm{~kg}^{-1}$ para $\mathrm{K}_{\mathrm{f}}$ do horizonte $\mathrm{A}$ e de 0,5 para o horizonte $\mathrm{B}$. Queiroz \& Lanças (1997) obtiveram um amplo intervalo de val ores de $K_{f}\left(0,81\right.$ a 5,75 $\left.\mathrm{L} \mathrm{kg}^{-1}\right)$ para 
solos de São Paulo, onde o teor de matéria orgânica ea CTC foram os atributos do sol o que mais afetaram a magnitude deste coeficiente.

O coeficiente de distribuição $K_{d}$ é usualmente calculado nos estudos de sorção de herbicidas, assim como se calcula o coeficiente de distribuição para um sistema líquido-líquido parcialmente misć́vel. Sendo assim, consideram-se as interações herbicidasolo não somente como um fenômeno de superfície, mas como um fenômeno que ocorre de maneira homogênea em todo o volume do solo $\left(K_{d}\right)$, ou da matéria orgânica $\left(K_{o c}\right)$. As regressões lineares que levaram ao cál culo de $\mathrm{K}_{\mathrm{d}}$ forneceram coeficientes de correlaçãor $>0,963$ ( $P<0,01$; dados não mostrados), o que valida a discussão desse coeficiente. Anal ogamente ao observado com o $\mathrm{K}_{\mathrm{f}}, \mathrm{oK}_{\mathrm{d}}$ foi menor na amostra oxidada (Quadro 2).

Os valores obtidos na normalização de $K_{d}$ em relação à superfície (K $\mathrm{K}_{\mathrm{d}} / \mathrm{ASE}$ ) (Quadro 2) mostram que a menor retenção de atrazina no solo após tratamento com peróxido não está relacionada com a diminuição da ASE total da amostra (Quadro 1), porém com a diminuição de sítios ativos por unidade de área superficial, ou seja, com a remoção do sorvente mais reativo. Este resultado corrobora a capacidade sorvente superior da matéria orgânica em relação aos componentes inorgânicos no solo, observada em outros estudos (Laird et al., 1994; Celis et al., 1997; M oreau et al., 1997). Herwig et al. (2001) obtiveram para caulinita pura o valor de $K_{d}$ de $50 \mathrm{~L} \mathrm{~m}^{-2}$, que corresponde a aproximada-mente 10 vezes o valor obtido na amostra oxidada do presente estudo. Esta diferença é devida provavelmente à composição heterogênea da amostra do Cambissolo e às diferentes condições experimentais empregadas nos dois estudos.

O val or de $K_{\text {oc }}$ obtido para o sistema sol o naturalatrazina (Quadro 2) encontra-se dentro do interval o de valores obtidos em solos por Queiroz \& Lanças

\section{Quadro 2. Parâmetros de Freundlich e coeficientes $K_{d}$ eK ${ }_{o c}$ para a sorção de atrazina em Cambissolo Húmico na forma natural e oxidada}

\begin{tabular}{lcc}
\hline \multirow{2}{*}{ Parâmetro } & \multicolumn{2}{c}{ Amostra } \\
\cline { 2 - 3 } & Natural & Oxidada \\
\hline $\mathrm{K}_{\mathrm{d}}\left(\mathrm{L} \mathrm{kg}^{-1}\right)$ & 4,64 & 0,33 \\
$\mathrm{~K}_{\mathrm{d}} /{\mathrm{ASE}\left(\mathrm{L} \mathrm{m}^{-2}\right)}$ & 46,4 & 4,4 \\
$\mathrm{~K}_{\mathrm{oc}}\left(\mathrm{L} \mathrm{kg}^{-1}\right)$ & 103 & $\mathrm{nc}$ \\
$\mathrm{K}_{\mathrm{f}}\left(\mathrm{L} \mathrm{kg}^{-1}\right)$ & 1,10 & 0,84 \\
$\mathrm{n}_{\mathrm{f}}$ & 1,40 & 0,78 \\
\hline
\end{tabular}

$\mathrm{nc}=$ não cal culado; $\mathrm{K}_{\mathrm{d}}$ : coeficiente de distribuiç̧ão; $\mathrm{K}_{\mathrm{d}} / \mathrm{ASE}$ : coe ficiente de distribuição normalizado por unidade de área; $\mathrm{K}_{\mathrm{OC}}$ : coeficiente de distribuição relativo ao carbono orgânico; $K_{f}, n_{f}=$ parâmetros de Freundlich.
(1997), 59 a $170 \mathrm{~L} \mathrm{~kg}^{-1}$, porém é inferior aos observados por Vaz et al. (1997), 357 a $591 \mathrm{~L} \mathrm{~kg}^{-1}$. O resultado de Koc caracteriza a atrazina comercial nesse sistema como um herbicida de alta mobilidade. Segundo Bewick (1994), herbicidas de mobilidade alta apresentam $50<\mathrm{K}_{\mathrm{OC}}<100 \mathrm{~L} \mathrm{~kg}^{-1}$. Não foi calculado o valor de $\mathrm{K}_{\mathrm{oc}}$ para a sorção na amostra oxidada, porque a quantidade de matéria orgânica neste sol o foi muito baixa (Quadro 1).

\section{CONCLUSÃO}

1. O model o de F reundlich mostrou-se adequado para avaliar a sorção da atrazina em Cambissolo Húmico, o que ficou evidenciado pelos altos valores de correlação obtidos. A matéria orgânica mostrouse um sorvente relevante na retenção de atrazina no solo, enquanto a contribuição dos componentes inorgânicos foi inferior.

2. O valor de $\mathrm{K}_{\mathrm{oc}}$ obtido classifica a atrazina comercial investigada como de alta mobilidade no sistema atrazina-solo estudado. A possível contaminação de lençóis freáticos e mananciais adjacentes a áreas deste tipo de solo submetidas à aplicação de atrazina deve ser monitorada.

\section{AGRADECIMENTOS}

Os autores agradecem à FAPERGS, pelo financiamento do projeto, e ao CNPq, pela concessão de bolsas de iniciação científica e de produtividade em pesquisa.

\section{LITERATURA CITADA}

ALBUQUERQUE, M.A.; SCHAEFER, C.E.G.R.; FOLONI, J.M.; KER, J.C. \& FONTES, L.E.F. Mineralização e sorção da atrazina em latossol o roxo sob cultivo convencional eplantio direto. R. Bras. Ci. Solo, 25:179-188, 2001.

ARMSTRONG, D.E.; CHESTER, C. \& HARRIS, J.H. Atrazine hydrolysis in soil. Soil Sci. Soc. Am. Proc., 31:61-67, 1967.

ATKINS, P.W. Físico-química, 6ed. Rio de J aneiro, Livros Técnicos e Científicos, 1999. v.2. 252p.

BARRIUSO, E. \& CALVET, R. Soil type and herbicides adsorption. Intern. J. Environ. Anal. Chem, 46:117-128, 1992.

BIRADAR, D.P. \& RAYBURN, A.L. Chromosomal damage induced by herbicide contamination at concetrations observed in public water supplies. J. Environ. Qual., 24:1222-1225, 1995. 
BELLUCK, D.A.; BOLAN, N.S.; RAHMAN, A. \& TILMAN, R.W. Groundwater contamination by atrazine and its metabolites: risk assessment, policy, and legal implications. In: SOMASUNDARAM, L \& COATS, J.R., eds. Pesticides transformation products: fate and significance in the environment. Washington, DC. American Chemical Society,, 1991. p.254-273.

BEWICK,D. The mobility of pesticides in soil - studies to prevent groundwater contamination In: BÖRNER., H. Pesticides in ground and surface water. Heidel berg, Springer-Verlag, 1994. p.61.

CELIS, R.; CORNEJ O, J.C.; HERMOSÍN, M.C. \& KOSKINEN, W.C. Sorption-desorption of atrazineand simazine by model soil colloidal componentes. Soil Sci. Soc. Am. J., 61:436443, 1997.

DICK, D.P.; GOMES, J .; BAYER, C. \& BODMANN, B. Adsorção de ácidos húmicos em latossolo roxo natural e tratado com oxalato de amônio. R. Bras. Ci. Solo, 24:285-294, 2000.

EMPRESA BRASILEIRA DE PESQUISA AGROPECUÁRIA EMBRAPA. CentroNacional dePesquisa de Solos. Sistema Brasileiro de Classificação de Solos. Rio de J aneiro, 1999. 412p.

EMPRESA BRASILEIRA DE PESQUISA AGROPECUÁRIA EMBRAPA. Centro Nacional de Pesquisas de Solos. Manual demétodos deanálises desolo. 2.ed. Rio deJ aneiro, 1997. 171p.

FARMER, V.C. The infra-red spectra of minerals. London, Mineralogical Society, 1974. 537p. (Monograph, 4)

GRAYMORE, M.; STAGNITTI, F. \& ALLISON, G. Impacts of atrazine in aquatic ecosystems. Environ. Intern., 26:483495, 2001.

J AVARONI, R.C.A.; LANDGRAF, M.D.; REZENDE, M.O.O. Comportamento dos herbicidas atrazina ealacror aplicados em solo preparado para o cultivo de cana-de-açúcar. Química Nova, 22:58-64, 1999.

HERWIG, U.; KLUMPP, E.; NARRES, H.D. \& MILAN, S.J. Physicochemical interactions between atrazine and clay minerals. Appl. Clay Sci., 18:201-222, 2001.

KRUGER, E.L.; RICE, P.J .; ANHALT, J.C. \& COATIS, J.R. Comparative fates of atrazineand deethylatrazine in sterile and non-sterile soils. J . Environ. Qual., 26:95-101, 1997.

LAGALY, G. Pesticide-day interactions and formulations. Appl. Clay Sci., 18:205-209, 2001.

LAIRD, D.A.; BARRIUSO, E.; DOUDY, R.H. \& KOSKINEN,W.C. Adsorption of atrazine of smectites. Soil Sci. Soc. Am. J., 56:62-67, 1992.

LAIRD, D.A.; YEN, P.Y.; KOSKINEM, W.C.; STEINEHEIMER, T.R. \& DOWDY, R.H. Sorption of atrazine on soil clay components. Environ. Sci. Technol., 28:1867-1873, 1994.
MARTIN-NETO, L.;VIEIRA, E.M. \& SPOSITO, G. Mechanism of atrazine sorption by humic acid: a spectroscopic study, Environ. Sci. Technol, 28:1867-1873, 1994.

MARTIN-NETO, L.; TRAGHETTA, D.G.; VAZ, C.M.P.; CRESTANA, S. \& SPOSITO, G. On the interaction mechanisms of atrazine and hydroxyatrazine with humic substances. J. Environ. Qual., 30:520-525, 2001.

MOREAU, C. \& MOUVET,C. Sorption and desorption of atrazine, deethylatrazine,and hydroxyatrazine by soil and aquifer solids. J. Environ. Qual., 26:416-424, 1997.

MEKARU, T. \& UEHARA, G. Anion adsorption in ferruginous tropical soils. Soil Sci. Soc. Am. Proc., 36:296-300, 1972.

PATLAK, M. Estrogens may link pesticides breast cancer: a controversial hytpothesis suggests that pesticides impact on estrogen metabolism can trigger cancer. Environ Sci. Technol., 30:210A-211A, 1996.

PICOLLO,A.; CONTE,P.;SHEURENERT,I. \& PAZI, M. Atrazine interactions with soil humic substances of different molecular structure, J. Environ. Qual., 27:1324-1333, 1998.

QUEIROZ, M.E.C. \& LANÇAS, F.M. HRGC Study of sorption and desorption of atrazine, ametryn and metolachor on Brazilian Soils. J. Braz. Chem. Soc., 8:1-6, 1997.

QUIRK, A. Significance of surface areas calculated from water vapour sorption isotherms by the use of the BET equation. Soil Sci., 80:423-430, 1955.

RODRIGUES, B.N. \& ALMEIDA, F.S. Guia de herbicidas, 4.ed. Londrina , Instituto Agronômico do Paraná, 1998. 675p.

SAWHNEY, B.L. \& SINGH, S.S. Sorption of atrazine by AI- and Ca- satured smectite, Clays Clay Miner., 45:333-338, 1997.

SENESI, N. \& TESTINI,C. Physico-Chemical investigations of interaction mechanisms between s-triazine herbicides and soils humic acids. Geoderma, 28:129-146, 1982.

SPARKS, D.L. Environmental soil chemistry. San Diego, American Press, 1995. 267p.

SPOSITO, G. The chemistry of soils. New York, Oxford University Press, 1989. 223p.

STEINBERG, C.E.W.; LORENZ, R. \& SPIEZER, O.H. Efects Brachydanio rerio. Water Res., 29:981-985, 1995.

TAN, H.K. I nfrared spectroscopy, Chapter 15. In: Soil sampling, preparation and analysis. New York, Marcel Dekker, Inc 1996. p.278-298.

VAZ, C.M.P.; CRESTANA, S.; MACHADO, S.A.S.; MAZO, L.H. \& AVACA, L.A. Adsorption isotherms for atrazine on soils measured by differential pulse polarography. Electroanalysis, 9:956-958, 1997.

XING, B.; PIGNATTELLO, J .J . \& GIGLIOTTI, B. Comparative Sorption between atrazine and other organic compounds in soils and model sorbents. Environ. Sci. Technol., 50:2432-2440, 1996 